\title{
The Application of 3D Model Retrieval Technique in Pipe Segment Merging Number
}

\author{
Zhao Hai-ying $^{1}$, Liu Jing-hua ${ }^{2}$ \\ (School of Mechanical Engineering and Automation, Beihang University, Beijing 100191, China) \\ ${ }^{1}$ zhaohaiying0423@163.com
}

Key words: Based on shape distribution, merge number, computer aided design, factory design

\begin{abstract}
This paper introduces the concept and basic framework of 3D model retrieval technology based on shape distribution, At the same time, according to the shape feature extraction and processing method of the model, the retrieval technique is introduced and applied in detail. This paper focuses on how to apply the 3D model retrieval technology based on shape distribution to the $\mathrm{CAD} / \mathrm{CAM}$, especially in the pipe segment merging number of the actual project. Today, with the rapid development of computer aided design, the application of this technology can meet the needs of the factory design, and it is also a breakthrough in the technical level. The application of 3D model retrieval technology based on shape distribution is a breakthrough attempt, although it has some technical problems, but it is of great significance.
\end{abstract}

\section{Introduction}

With the further development of three-dimensional technology, three-dimensional model retrieval technology in people's daily life as well as CAD/CAM computer animation design has been more and more applications, At the same time it has become a very important part of the MPEG7 standard model [1],the 3D model shape distribution retrieval technology to break the usual keyword retrieval method based on the limitation, can be directly used to shape 3D model and topological index and complete the follow-up retrieval task.

Three dimensional factory design is an important application field of computer aided design, along with the development of Engineering Technology, the number of pipe segment is greatly increased in the field of petroleum, chemical engineering and other areas of the factory design. The accuracy of the pipe section determines the effect of the final project, using the computer aided design technology to carry out the pipe section and the combination number can improve the work efficiency and reduce the construction cost.

The development and current situation of the 3D model retrieval technology based on shape feature and the pipe segment and merge number are studied, the paper mainly discusses the application of 3D model retrieval technology based on shape feature in the design of pipe segment merging number in 3D factory, and the application of 3D model retrieval based on shape feature in computer aided design has been made.

\section{Summary of 3D retrieval technology based on shape distribution}

\subsection{Concept introduction of 3D retrieval technology based on shape distribution}

Content based 3D model retrieval can be broadly divided into three categories: (1) Technology based on shape feature; (2) Technology based on Topology (mainly used for the extraction of 3D skeleton [2]); (3) Technology based on image comparison. The 3D model retrieval based on shape feature is an important technique to retrieve the key technology.

\subsection{Shape based 3D model retrieval system}

In the recent research field, the shape based 3D model retrieval is a new direction, and its idea is derived from the content based image retrieval. Although the field would like to be active, but there is still a problem to be solved only one step. 


\subsubsection{General framework of 3D shape retrieval system}

Figure 1 is a typical system framework for 3D shape retrieval, which includes an online search engine and a database with index structure, which is created in the offline state.

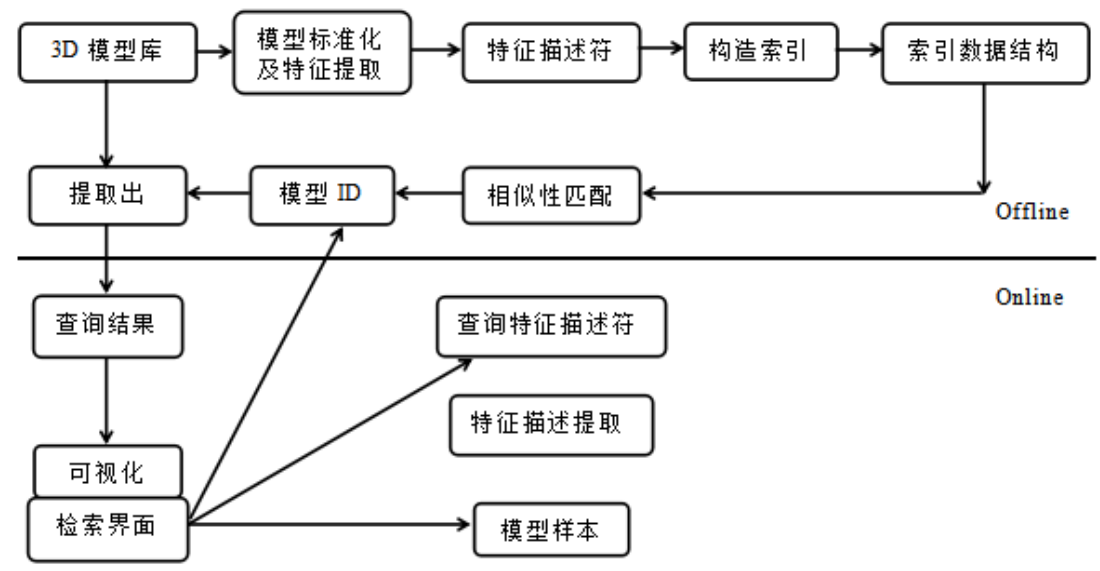

Fig.1 The Overall Framework of 3D-model Retrieval

\subsubsection{Basic process of 3D model retrieval algorithm}

The shape information of the 3D model is mapped onto the feature space, which is the main idea of shape retrieval, and by acquiring the relevant feature vectors, compare the obtained feature vector value to complete the model retrieval. Retrieval algorithm is generally divided into three steps: (1) model standardization; (2) shape feature extraction; (3) similarity measure. The core problem is how to extract the model features.

Model standardization

In this paper, we mainly introduce two typical methods of extraction, which can ensure the rotation invariance of the extracted features:

(1) Principal component analysis (component analysis principal, PCA) [3], is a kind of information processing, compression and extraction and based on the effective method of covariance matrix.

(2) Spherical harmonic expression

The spherical harmonic expression is represented by the harmonic function. The spherical harmonic function is the solution of the Laplasse equation in the spherical coordinates. The spherical harmonic function can be used, and the corresponding spherical function is constructed according to the characteristics of the model [4], the characteristic of model retrieval is constructed by using the expansion coefficients of different frequency.

Shape feature extraction

The significance of feature extraction is to extract the factors which can reflect the difference of different 3D models. The matching method of 3D model is to use the feature descriptor to describe the model features, and to achieve the goal of the retrieval model. Therefore, the feature descriptor should choose suitable for model categories, the feature extraction method of concrete will be detailed in the second part.

Similarity measurement

Similarity measurement is to determine whether the two dimensional model is similar by calculating the distance of the feature descriptor in the feature space of the two models. The similarity measure defines the distance of the feature descriptor in the feature space, which is usually defined as four properties, which are symmetry, difference, identity and transform invariance. Four specific meaning properties vary greatly, symmetry and not in all cases, it is generally regarded as the nature of a concept hierarchy. The difference indicates that the different shapes are not exactly alike; the identity is that any shape can be exactly matched with one's own. If the shape descriptor has nothing to do with the orientation, scale and direction of the model, the transform invariance of the distance definition must possess. 


\section{Application of 3D retrieval based on shape distribution in pipe segment merging number}

In the field of CAD/CAM, the research and application of 3D CAD model retrieval technology is still at the initial stage at home and abroad, and there is almost no application in the combined number of the pipe segment in the factory model (Figure 2).At present, most of the 3D model retrieval methods used in the CAD model are used in the general field, other is to take into account the CAD model of some special data and mechanical design and manufacture of specific information and knowledge in the field of specific design of the new method is not perfect. Therefore, access to a large number of documents, and try to use the 3D retrieval technology based on shape distribution to complete the similarity comparison and merge number of the pipe segment model. Next, this paper introduces the application of the technology in the factory model:

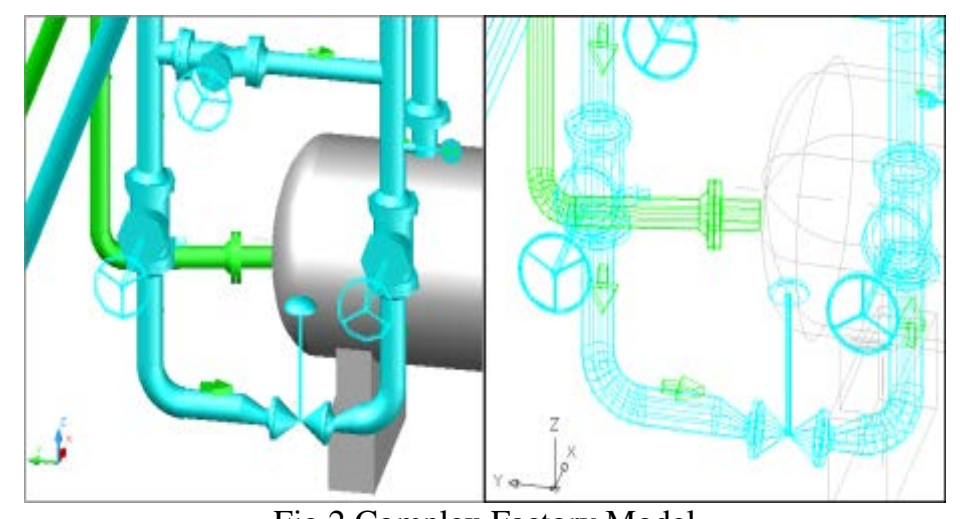

Fig.2 Complex Factory Model

3.1 Concept of pipe section and merging number in factory model

In this discussion is the pipe segment, pipe section and pipe are different. In the pipe, classification is very clear, pipe fitting (including flange, three links, etc., as shown in Figure 3)is a general designation of parts and components that are connected, controlled, changed to, split, sealed, supported, and so on. The pipe section is defined as the pipe section, which is a combination of two or more pipes.

The number and size of the pipe section must be clearly defined after the pipe section is fabricated. The merging number of pipe segments is actually to belong to the same pipe section, which is the same as the pipe section, will no longer be a separate number, thereby reducing production costs, to further improve the production efficiency [6].

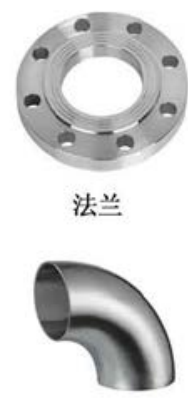

弯头
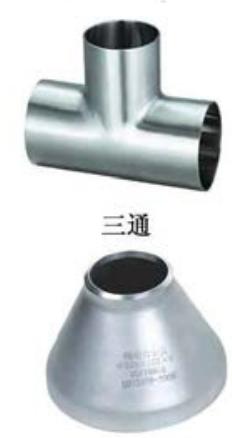

异径管

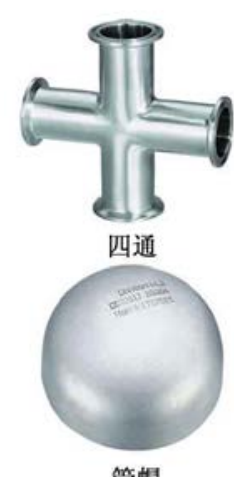

管帽

\subsection{Standardization of fitting model}

Fig.3 All Kinds of Pipe Fittings

This section of the model is reduced to pipe segment extraction. In addition, we briefly introduced the data structure of our factory model, so as to further understand the extraction algorithm. In the factory model, each entity group describes a specific information of a component, 
which is composed of a number of entities. All components are composed of two entities: the intelligent line entity group (group SMRTLN_BEGIN) and the model diagram entity group (group MODEL_BEGIN), which is used to store the engineering parameters and geometric parameters. Each entity group of the same element forms a one-way circular linked list by the group of the group (shown in Figure 4).

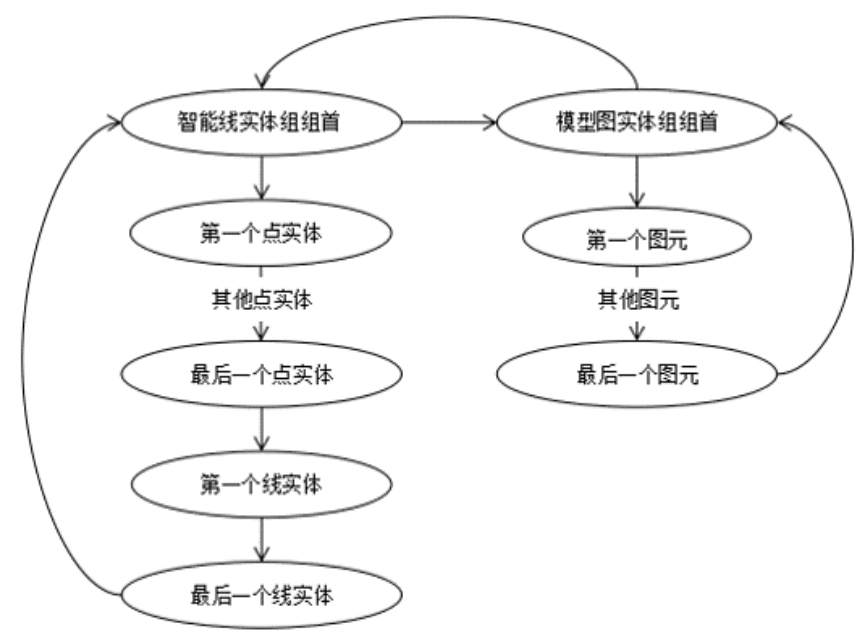

Fig.4 Component Structure Diagram

3.3 Shape feature extraction of pipe segment model

Features can be divided into two categories according to their representation: topology and feature vector.In view of the complexity and variety of the connection mode of the pipe section model,more obvious characteristics of spatial characteristics, we choose method of skeleton map in topology structure as its shape feature expression method. Sundar et al. Prior to the proposed framework based on the method of [7] to extract the characteristics of the model,calculation of the model skeleton nodes, and through each node to construct the corresponding skeleton map, and the pipe section model just in line with this requirement, the pipe section of its own structure is equivalent to the non circular graph.

3.4 Shape similarity matching and number of pipe segment model

\subsubsection{Precast pipe section}

Prior to the shape similarity matching of the pipe segment model, the pipe segment is first needed to be prefabricated. To draw a good one or more lines according to the user's requirements are broken into different pipe segments. The number of pipe fittings in the pipe is determined by the structure of the pipeline. The break point for the division of the pipe is prefabricated solder joint, that is, the connection of some pipe fittings will automatically add the solder joints as the demarcation point of the pipe section (generally meet the flange port will be disconnected for the pipe section).The rule of the prefabricated pipe section includes: the end point of the precast pipe segment is the end point or the non prefabricated solder joint; all the pipe fittings in the prefabricated pipe section belong to the same pipeline number.

Depth first traversal algorithm is adopted in the pipe segment (Fig. 5): 


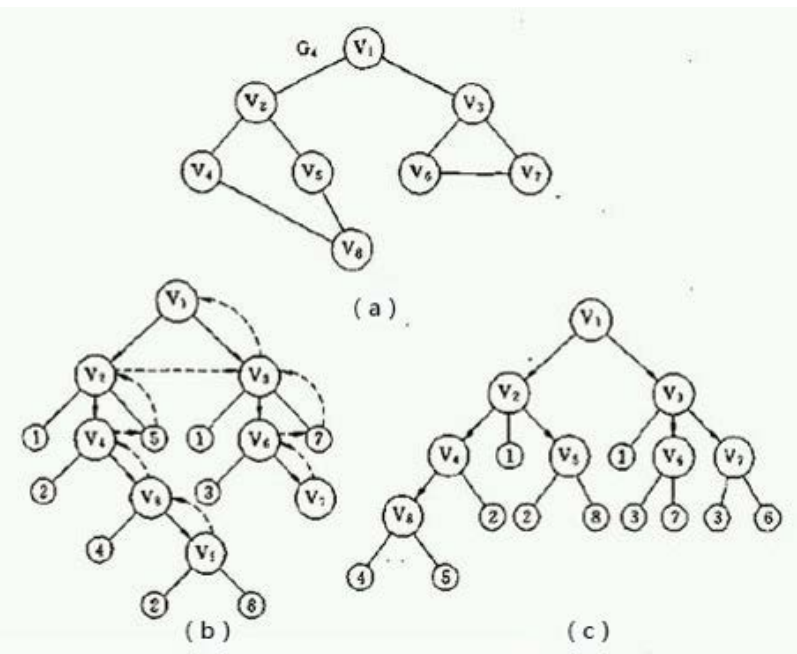

Fig.5 Sample of Topology

Specific algorithm processes are as follows (Fig. 6):

1) All SMRTLN_BEGIN in the model group the first intelligent.

2) Either take an object (not already in the selection of centralized processing) began to be connected to traverse, where to traverse the pipe into the selection has been processed.

3 ) The use of prefabricated pipe traversal operator, from the first test object traversing, each traversal obtained is a prefabricated pipe section.

4) Each traverse also has to get the edge of the pipe, from the edge of the pipe loop, until the end of the connection.

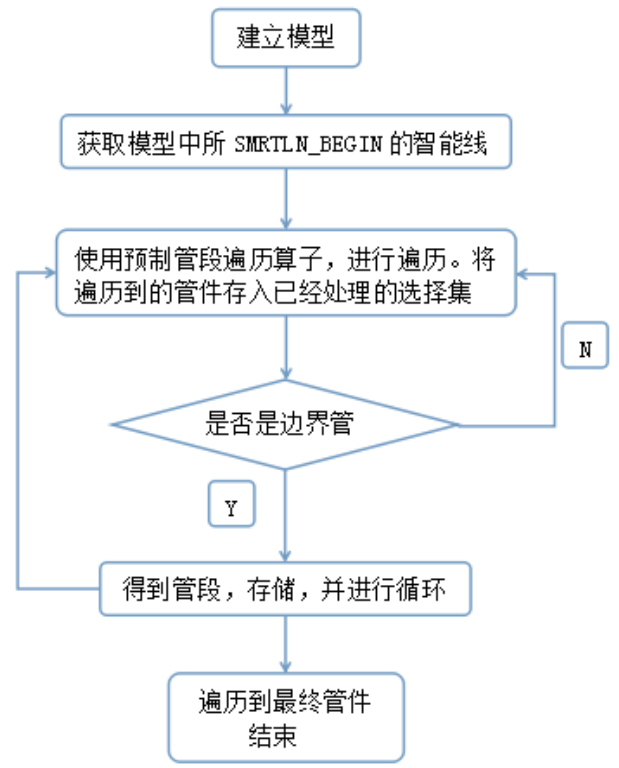

3.4.2 Tube segment similarity measure

Fig.6 Algorithm Flow Diagram of Extracting Pipe

1) Measurement method selection

At present, in view of the shape based 3D model retrieval, there are two kinds of matching methods: distance measure method and classification method. In view of the characteristics of the pipe section and the applicability of the two methods, this method is used to extract and compare the distance measure which is suitable for the similarity measurement of pipe segment.

The distance measure method is more common in the model matching method.By comparing $t$ he shape of et.al.[8] Osada and the shape of the model, it is proved that the Euclidean distance of sh ape distribution is the most effective method to measure the properties of random surface points.At $t$ he same time, the application of et.al.[9] Ip to the shape distribution in the CAD and solid modeling, the Euclidean distance is redefined,moreover, it is very useful for the entity model. Therefore, the $\mathrm{E}$ uclidean distance is used to measure the pipe segment model. 
2) Similarity measure

In the previously mentioned model of intelligent line list, the coordinates of the starting point of each pipe in the pipe section are stored, and various kinds of pipe fittings, number and other information are also stored. Therefore, the initial measurement of the type and number of pipe segments included in the pipe segment can be carried out first. In the case of the same type and number of known pipe fittings. The direction vector of each tube in the tube can be calculated through the $\mathrm{X}$ and $\mathrm{Y}$ coordinates of the starting point, and then the three-dimensional coordinate system can be constructed.

Starting point for the starting point of the order of extension:

1. The pipeline direction of the starting point as the $\mathrm{X}$ direction, the first branch or the turning point of the Y direction, after the Euclidean distance formula was formed, the UCS was formed;

2. All nozzle coordinates out of the starting point alternate the above established UCS;

3. If all the nozzle UCS coordinates, then the relative space position, otherwise the pipe is not the same.

\subsubsection{Numbering principle and number}

The difference between different pipeline is required, that is, the prefix is different from the number of pipe segment in different pipeline. The same pipe segment number prefix is the same in the same pipeline, by comparing the method of establishing the coordinate system, different pipe sections are numbered according to predetermined rules, so as to facilitate the differentiation between different segments.

After the numbering of corresponding numbering rule is completed, the number of each pipe segment is stored in the linked list of the pipe segment itself, so the function of the number of pipe section is successfully completed.

\section{Conclusion}

Through the brief introduction of the technology of 3D model retrieval based on shape distribution, it can be applied to the pipe section and the number of the engineering model effectively, finally, the engineering model can be extracted from the pipe section, and the similarity comparison, given the corresponding number. It promote the great progress of the factory design, but also a new breakthrough in computer aided design.

The 3D model retrieval is a new research direction in the field of computer graphics in recent years, although it is in the initial stage, but with the wide application of the technology, especially for the first time, the technology used in the pipe section of the merger number, is bound to promote the engineering application of technological change. Therefore, the three-dimensional model retrieval technology in the application of CAD challenges will be more and more, we should be prepared to overcome more.

\section{References}

[1] Yang Yu-bin, Lin Jun, Zhu Qing. A survey of 3D model retrieval based on shape features [J]. Chinese Journal of Computers, 2004, 27 (10) : 1297-1310.

[2] Xu Shi-Biao, Che Wu-Jun, Zhang Xiao-Peng. A survey of 3D model retrieval technology based on shape features. Chinese Journal of Stereology and Image Analysis 2010, 4 439-450.

[3] Pottmann H, Leopoldseder S, Hofer M. Approximation with active b-spline curves and surfaces[C]//Pacific Graphics. Beijing: IEEE Computer Society, 2002:8-25.

[4] Pascucci V, Scorzelli G, Bremer P T, et al. Robust on-line computation of reeb graphs: simplicity and speed [J]. ACM Transactions on Graphics, 2007, 26(3): 581-589.

[5] Attene M, Biasotti S, Mortara M, et al. Computational methods for understanding 3D shapes [J]. Computer Graphics, 2006,30 (3): 323-333.

[6] Zhu Jun-Fei, Zhang Jing-Fen. The factory prefabrication of pipes and its application examples. Chemical Production and Technology 2015, 4,57-60 
[7] Du H X, Qin H. Medial axis extraction and shape manipulation of solid objects using parabolic PDEs[C]// ACM Symposium on Solid Modeling and Applications. Genoa: ACM, 2004:25-34.

[8] Osada R, Funkhouser T, Chazelle B, et al. Shape distributions [J]. ACM Transactions on Graphics, 2002,21 (4): 807-832.

[9] Ip C Y, Lapadat D, Sieger L, et al. Using shape distributions to compare solid models[C]// ACM Symposium on Solid and Physical Modeling. Proceedings of the seventh ACM symposium on Solid modeling and applications, Saarbrücken, Germany: ACM, 2002: 273-280.

[10] Cui Chen-Yang,Shi Jiao-Ying.Analysis of Feature Extration in 3D Model Retrieval. Journal of Computer-Aided Design \& Computer Graphics 2004, 7,882-889. 